\title{
BRCA1 Positivity in Triple Negative Breast Cancers: A Prospective Study in Tertiary Care Cancer Hospital
}

\author{
Sharma $M^{1}$, Madan $M^{2}$, Manjari $M^{3}$, Madhukar $M^{4}$, Singh $T^{5}$, Gupta $S^{6}$ \\ ${ }^{1}$ Dr Manisha Sharma, Associate Professor, ${ }^{2}$ Dr Manas Madan, Associate Professor, ${ }^{3}$ Dr Mridu Manjari, Professor and \\ Head, ${ }^{4}$ Dr Mohit Madhukar, Resident, ${ }^{5}$ Dr Taranveer Singh, Resident, ${ }^{6}$ Dr Shruti Gupta, Resident; all authors are \\ attached with Department of Pathology, SGRDIMSAR, Amritsar, Punjab India
}

Address for Correspondence: Dr Manisha Sharma, B-241, Ranjit Avenue, Amritsar, SGRDIMSAR, Amritsar. manisha_salwan@yahoo.com,

\begin{abstract}
Objectives: In the present study we investigated the BRCA1, Estrogen receptors status (ER), progesterone receptor status (PR) and human epidermal growth factor receptor (Her2neu) expression in selected patients with higher probability of BRCA1 positivity. The association of BRCA1 positivity and Triple Negative Breast Cancer (TNBC) status was also demonstrated. Materials and Methods: The study was conducted on 100 cases of breast cancer specimen received in department of Pathology, SGRDIMSAR, Amritsar. Histopathological typing and grading was done followed by immunohistochemistry for BRCA1, ER, PR and Her2neu. Results: All the tumors were infiltrating ductal carcinoma NOS. 4 cases were of grade I, 34 cases as grade II and 62 as grade III. ER+ PR+ Her2neu-ve cases comprised of $33 \%$ and triple negative being $38 \%$. BRCA1 positivity was seen in $36 \%$ patients. BRCA1 positivity was significantly higher in triple negative primary tumours as compared to other groups. (23/38- $60.5 \%$ vs 13/62-20.9\% respectively) with significant p - 0.009. Conclusion: Triple Negative Breast Cancer (TNBC) patients are associated with poor prognostic factors and are more likely to be BRCA1 positive. Such BRCA1 identification is not only important in management of the patient for contralateral breast cancer and ovarian cancer but also blood relatives of such patients. As the relatives of such patient have $50 \%$ of increased chances of BRCA1 expression.
\end{abstract}

Keywords: Triple negative Breast Cancer, BRCA1, ER, PR, Her2neu

\section{Introduction}

Breast Cancer is one of the leading cause of mortality in middle aged women. In the year 2000, 796,000 new breast cancers were diagnosed and it accounted for 314,000 deaths worldwide [1,2]. Among familial breast cancers BRCA1 and BRCA2 were estimated to be responsible for $75 \%$ of the cases. However, recent studies have proved this percentage to be less and varying depending upon the population studied [2]. According to recent studies, BRCA1 gene mutations had been found associated with increased life time risk of carcinoma breast of $40-57 \%$ and an $18-40 \%$ life time risk of ovarian malignancy [3]. Such patients also show increased risk of contralateral breast carcinoma of about $50 \%$ after 25 years of diagnosis [4,5]. It has been analysed that the cancer arising in BRCA1 mutation carriers is different from non BRCA1 mutation carriers.

Manuscript received: $4^{\text {th }}$ August 2016

Reviewed: $14^{\text {th }}$ August 2016

Author Corrected: $26^{\text {th }}$ August 2016

Accepted for Publication: $13^{\text {th }}$ September 2016
BRCA1 mutation positive cases have higher grades with poorly differentiated morphology, higher mitotic count and pleomorphism [3,4,5].

The choice of therapeutic regimens for breast cancer patients depends upon the various predictive and prognostic factors as grade of the tumour, stage of the tumour, oestrogen receptor status (ER), progesterone receptor status (PR), human epidermal growth factor receptor 2 (Her2neu) and proliferative index. Hormone receptor status is prognostic and predictive factor as well predicting the response to various treatment plans [6,7]. Classification of breast carcinoma according to immunohistochemistry are luminal A (ER +ve and PR +ve, Her2neu -ve), luminal B (ER+ve, and/or PR +ve, Her2neu +ve or-ve), triple negative or basal like (ERve, PR-ve and Her2neu-ve) and Her2 type (ER-, PR-, Her2neu+). Triple negative breast cancers (TNBC) are the cancers which are negative for ER, PR and Her2neu 


\section{Research Article}

expression. Many features of such cases may overlap with basal like cancers but basal like classification involves immunophenotypic expression of cytokeratins and gene assays as well [8]. Though the response to chemotherapy in TNBC patients is good but such patients carry a poorer prognosis with higher risk of relapse and shorter period of disease free survival [9].

The prevalence of BRCA1 carriers among TNBC patients is more variable than triple negative pevalance among BRCA1 mutation carriers. Many studies have estimated the higher possibility and likelihood of BRCA1 mutation among TNBC patients. Such mutation identification is not only important in guiding the treatment of the patient but also the risk management in their blood relatives [10].

In the present study we investigated the BRCA1, ER, PR and Her2neu expression in selected patients with higher probability of BRCA1 positivity- patients with positive family history, young age of presentation, history of ovarian and contralateral breast cancer. The association of BRCA1 positivity and TNBC status was also demonstrated.

\section{Material and Methods}

The study was done on 100 cases of breast cancer received as mastectomy or lumpectomy in department of Pathology, SGRDIMSAR. Detailed clinical data was recorded as per the proforma attached. Cases with positive family history, younger age of presentation, history of contralateral breast cancer and ovarian cancers (cases which were more likely to be associated with BRCA1 positivity) were included in the study. The tissue was formalin fixed and paraffin embedded, then stained for hematoxylin and eosin for histopathological typing and grading. The histopathological grading of the breast carcinoma was done according to the Nottingham modification of Bloom Richardson grading system [11]. All the cases were subjected to immunohistochemistry for ER, PR, Her2neu and BRCA1 expression. Immunohistochemistry was performed by using antibodies against the estrogen receptors (ER), the progesterone receptors (PR), Her2neu receptors (Diagnostic Biosystem) and BRCA1 (Biocare Medical). 3-5 $\mu \mathrm{m}$ sections were cut and mounted on poly-l-lysine coated slides. Slides were dried overnight at 37 degree and dewaxed in xylene and hydrated. The antigen retrieval was done by using pressure cooker method with $10 \mathrm{mmol}$ citrate buffer at $\mathrm{pH}$ 6. Endogenous peroxidase was neutralized using peroxidase block for 5 minutes. Tris buffer was used as the wash buffer. After protein blocking, the slides were incubated overnight with the available ER, PR, Her2neu and BRCA1 primary antibodies in moist chamber. After washing and applying the post primary block, sections were conjugated with streptavidin Horse Radish Peroxidase (HRP). The slides were covered with Diaminobenzene tetrahydrochloride (DAB) used as the chromogen, washed and counterstained with hematoxylin and were examined by light microscopy. $\geq 10 \%$ nuclei stained brown were taken positive for ER and even $1 \%$ stained were taken positive for PR. For BRCA1 this value for positive stained nuclei was $\geq$ $30 \%$. For Her2neu membranous staining in $>25 \%$ cells was taken as positive.

\section{Results}

Age of the patients varied from 23-70 years with the maximum number of the patients (57\%) belonging to age group of 41-60 years. Right side was slightly more commonly involved and upper outer quadrant being most commonly involved

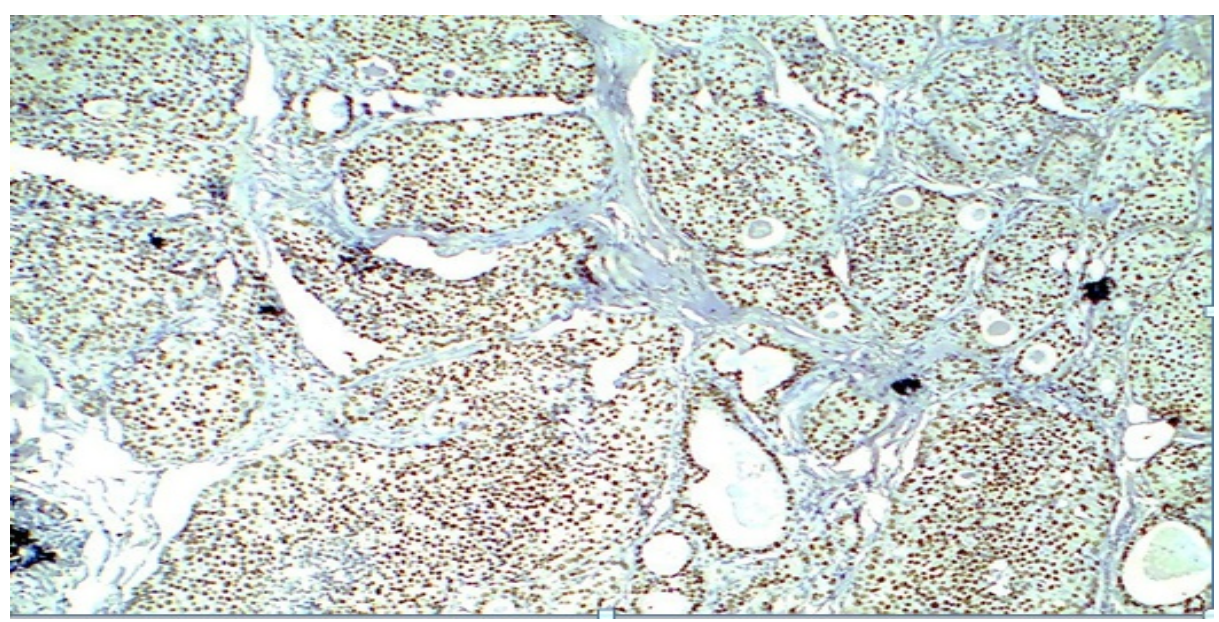

Fig (A): Immunohistochemistry showing ER positivity (Nuclear) - IHC (100X) 
site $(65 \%)$. The size varied from $1.5-4 \mathrm{cms}$ with maximum number of cases being in group of $2-5 \mathrm{cms}(70 \%)$. All the tumors were infiltrating ductal carcinoma NOS (Not Otherwise Specified). Grade III were maximum (62 cases) followed by 34 cases of grade II and 4 cases as grade I. Lymph nodes were recovered in 84 cases of radical mastectomy, Out of which 50 cases showed metastatic carcinomatous deposits. On immunohistochemistry, 36 cases showed BRCA1 positivity (Fig C) and ER PR positivity (Fig A, B) was observed in 35\% of the cases. 33 cases showing ER PR positivity were Her2neu negative. Only two cases were triple positive. Triple negative cases accounted for $38 \%$ of total while ER ve PR-ve and Her2neu +ve (Fig D) cases were $27 \%$.

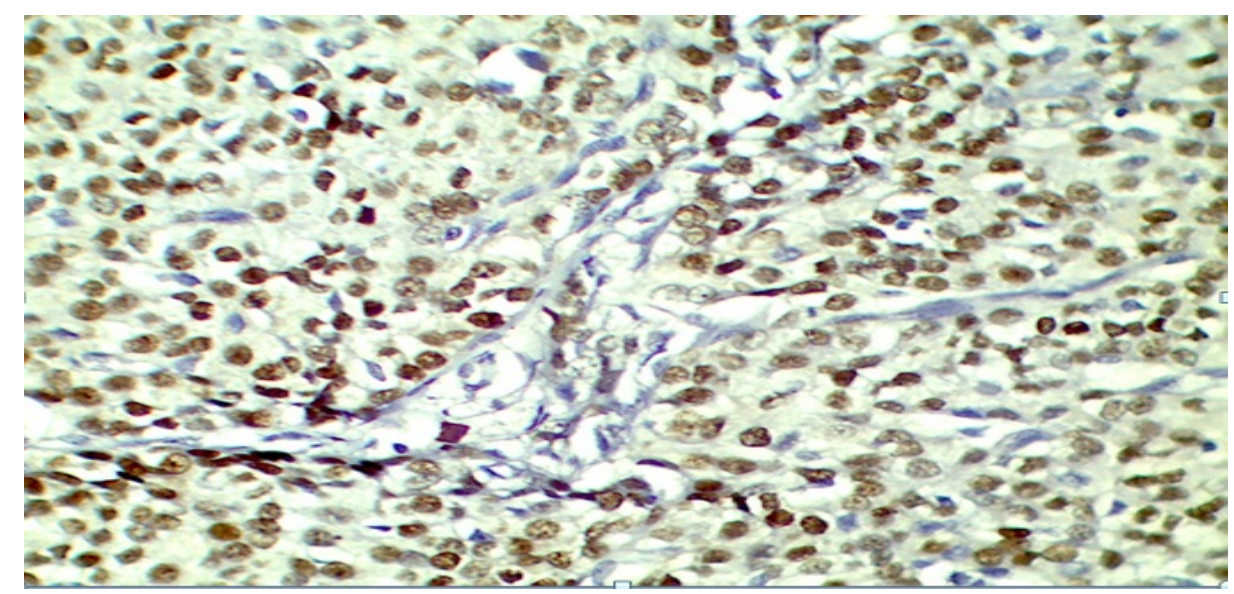

Fig (B) : Immunohistochemistry showing PR positivity (Nuclear) - IHC (400X)

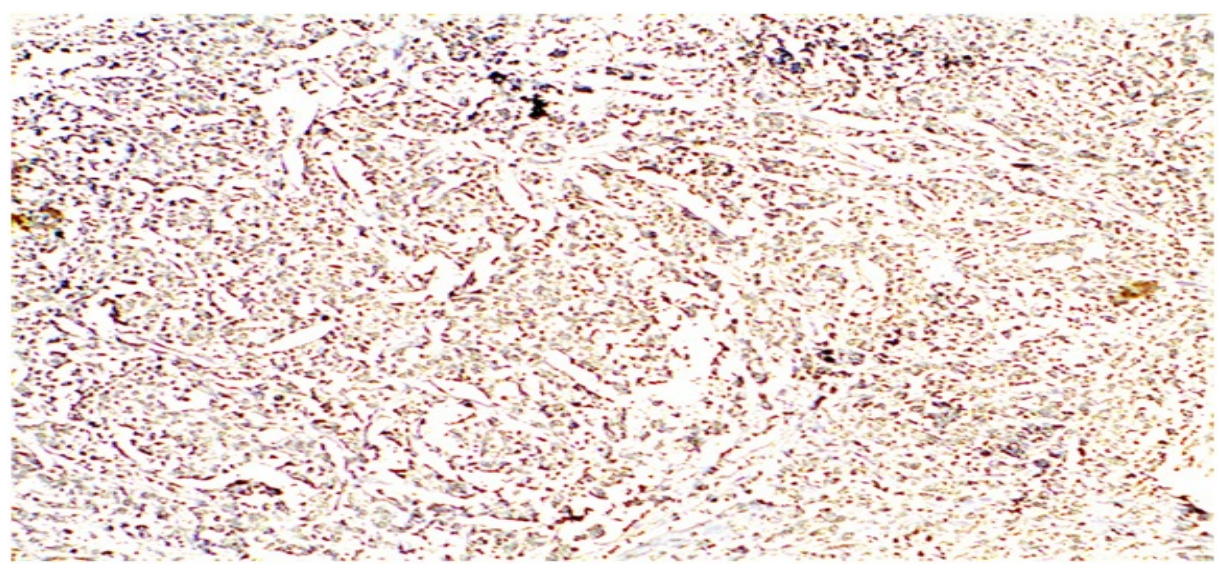

Fig (C): Immunohistochemistry showing BRCA1 positivity (Nuclear) - IHC (100X)

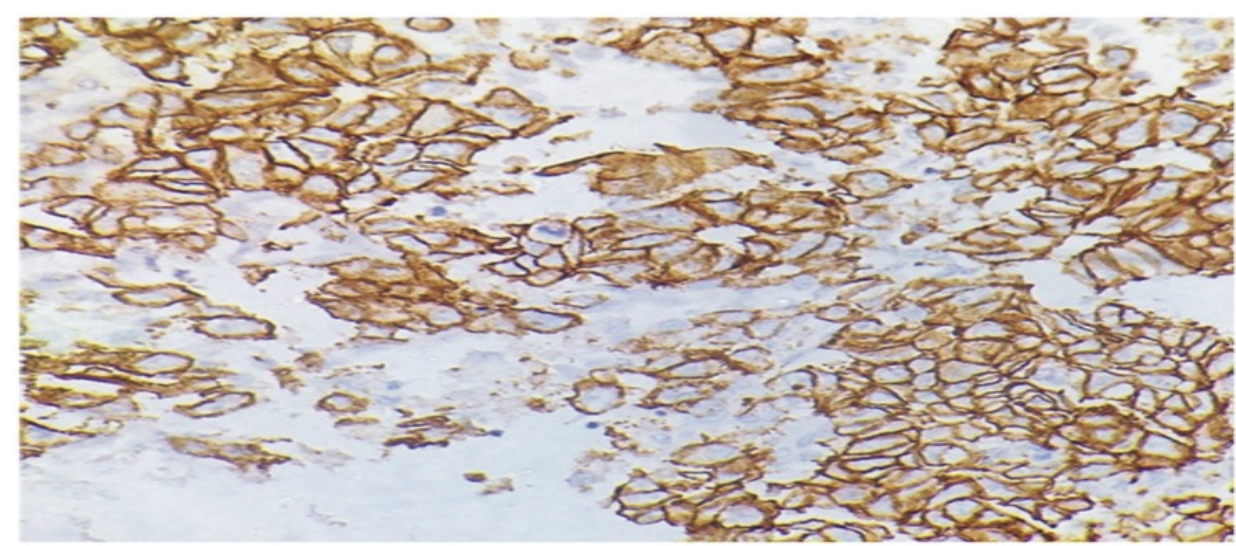

Fig (D): Immunohistochemistry showing Her2neu positivity (Membranous) - IHC (400X) 
BRCA1 expression was evaluated with the age of the patients, $63.8 \%$ of total BRCA1 positive cases belonged to younger age group of $<40$ years. (Table 1) BRCA1 positivity was significantly correlated with higher grade $(\mathrm{p}=0.013)$, lymph node metastasis $(\mathrm{p}=0.001)$ and larger tumour size $(\mathrm{p}<0.001)$. In contrast ERPR expression was associated with favourable prognostic markers as lower grade, smaller tumour size and no metastasis in lymph nodes.

Table-1: Distribution of patient according to BRCA 1 Mutation status and age at diagnosis.

\begin{tabular}{|c|c|}
\hline AGE (Years) & BRCA 1 positive cases \\
\hline $21-30$ & $12(33.33 \%)$ \\
\hline $31-40$ & $11(30.56 \%)$ \\
\hline $41-50$ & $4(11.11 \%)$ \\
\hline $51-60$ & $5(13.89 \%)$ \\
\hline $61-70$ & $4(11.11 \%)$ \\
\hline
\end{tabular}

Table-2: Correlation of BRCA1 positivity with Estrogen Receptor (ER), Pogesterone (PR) and Her2neu status including Triple Negative cases.

\begin{tabular}{|c|c|c|c|}
\hline ER, PR, Her2Neu status & BRCA 1 Positive & BRCA 1 negative & Total \\
\hline ER+, PR+, Her2Neu- & 5 & 28 & 33 \\
\hline ER-, PR-, Her2Neu+ & 7 & 20 & 38 \\
\hline ER-, PR-, Her2Neu- & 23 & 15 & 02 \\
\hline ER+,PR+, Her2Neu+ & 01 & 01 & 38 \\
\hline
\end{tabular}

Triple negative cases also showed positive correlation with poor clinicopathological parameters like grade, size and lymph node metastasis. On focusing the study on correlation of TNBC patients with BRCA1 positivity it was observed that $23 / 38(60.5 \%)$ BRCA1 positive cases belonged to triple negative breast cancers as compared to $13 / 62(20.9 \%)$ BRCA1 positive cases which were seen in other groups. Therefore it was concluded that BRCA1 positivity was significantly higher in triple negative primary tumour as compared to other groups with $\mathrm{P}=0.009$. (Table 2)

\section{Discussion}

Breast cancer is becoming number one cancer in Indian population like western world thus making cervical cancer as second common [12]. The aggressive biological behaviour of breast cancer is mostly the result of changes in molecular characteristics of tumor cells including alterations in mechanisms controlling adhesion, growth and proliferation.

A wide variety of morphology based and molecular based breast cancer prognostic factors and tumor markers had been studied to identify the oncogenes involved in initiation and progression of tumours and development of new anticancer drugs.

Earlier BRCA1 and BRCA2 were estimated to be responsible for $75 \%$ of familial breast cancers. Recent data shows this percentage to be less and depends upon population studied [2]. Immunohistochemical expression of BRCA1 protein has been studied as inexpensive and valuable preliminary method for detecting BRCA1 status with high sensitivity and specificity of anti-BRCA1 antibodies. BRCA1 positivity indicates the full length functional protein while negativity may be seen in sporadic BRCA1 mutation $[13,14]$. In the present study 36 cases showed immunopositivity for BRCA1. This high positivity was due to inclusion of cases with high probability of BRCA1 positivity in the present study (Cases with positive family history, younger age of presentation, history of contralateral breast cancer and ovarian cancers.) BRCA 1 expression was significantly correlated with higher grade of tumor $(p=0.013)$.

Similar results have been seen in various previous studies which have shown that BRCA1 related cancers were of higher grade $[15,16]$. BRCA1 positive cases had higher number of lymph node involvement and tumor size $>2 \mathrm{~cm}$ at the time of presentation. These results are in concordance with results of various researches $[16,17]$.

In our study significant correalation was found between BRCA1 positivity and younger age of presentation, $63.8 \%$ of total BRCA1 positive cases belonged to younger age group of $<40$ years corroborating the findings of previous studies where this percentage varied from 52-62\% [10,18]. 
In present study, ER PR positivity was reported in $35 \%$ of the cases which is bit lower than the percentage found in western world. ERPR expression was found to be associated with favourable prognostic markers as lower grade, smaller tumour size and no metastasis in lymph nodes. This result was consistent with previous reports $[19,20]$.

$38 \%$ cases accounted for Triple negative cases as compared to $20-35 \%$ in other studies $[19,20]$. TNBC showed positive correlation with poor clinicopathological parameters like grade, size and lymph node metastasis similar to the studies conducted in the past $[10,18,19]$. TNBC patients though respond well to chemotherapy but such patients have poorer prognosis because of higher risk of relapse and poorer overall outcome [9].

On demonstrating the correlation of TNBC patients with BRCA1 positivity it was concluded that BRCA1 positivity was significantly higher in triple negative primary tumour as compared to other groups. (23/38$60.5 \%$ vs $13 / 62-20.9 \%$ respectively) with significant $p$ value of 0.009 . Similar significant correlations had been shown by other researchers in their studies with $\mathrm{p}$ value ranging from 0.012-0.001[10,18].

The treatment approach in treating TNBC patients is different than those of hormonal receptors positive patients. Such patients require intensive treatment and carry poorer prognosis. Our study proved that TNBC is significantly associated with BRCA1 positivity thus demonstrating the effect of genetic background on phenotypic presentation of breast cancer. This correlation warrants the selection of TNBC patients for BRCA1 analysis specially in young patients as, such patients need extensive therapy and are at the higher risk of development of contralateral breast cancer and ovarian cancer.

\section{Conclusion}

TNBC patients are associated with poor prognostic factors and are more likely to be BRCA1 positive. Such BRCA1 identification is not only important in management of the patient for the risk of contraletral breast cancer and ovarian cancer but also in blood relatives of such patients. As the relatives of such patient have $50 \%$ of increased chances of BRCA1 expression. Further studies are required to determine whether treatment outcome in TNBC patients with BRCA1 positivity differs as compared to TNBC patients without BRCA1 mutation.
Funding: Nil, Conflict of interest: None initiated, Permission from IRB: Yes

\section{References}

1. Szabo CI, King MC. Population genetics of BRCA1 and BRCA2. Am J Hum Genet. 1997;60(5):1013-20.

2. Clays-Tagoe BNL, Yarney J, Kenu E, Amanhayian ONAK, Enchill E, Obeng I. Profile of cancer patients seen at Korle Bu teaching hospital in Ghana (A cancer registry review). BMC Research Notes. 2014;7:577. DOI:10.1186/1756-0500-7-577.

3. Chen S, Parmigiani G. Meta-analysis of BRCA1 and BRCA2 penetrance. J Clin Oncol. 2007 April 10; 25 (11):1329-33. DOI:10.1200/JCO.2006.09.1066.

4. Graeser MK, Engel C, Rhiem K.. Contralateral breast cancer risk in BRCA1 and BRCA2 mutation carriers. J Clin Oncol. 2009 Dec 10;27(35):5887-92. DOI:10. 1200/JCO.2008.19.9430.

5. Gronwald J, Tung N, Foulkes WD. Tamoxifen and contralateral breast cancer in BRCA1 and BRCA2 carriers: an update. Int J Cancer. 2006 May 1;118(9): 2281-4. DOI:10.1002/ijc.21536.

6. Fuksa L, Micuda S, Grim J, Ryska A, Hornychova H. Predictive biomarkers in breast cancer: their value in neoadjuvant chemotherapy. Cancer Invest. 2012; 30:663-78.

7. Thibault C, Khodari W, Lequoy M, Gligorov J, Belkacémi Y. HER2 status for prognosis and prediction of treatment efficacy in adenocarcinomas: A review. Crit Rev Oncol Hematol. 2013;88:123-33.

8. Dawson SJ, Provenzano E, Caldas C. Triple negative breast cancers: clinical and prognostic implications. Eur J Cancer. 2009 Sep;45(1):27-40. DOI:10.1016/SO9598049(09)70013-9.

9. Metzger-Filho O, Tutt A, de Azambuja E, Saini KS, Viale G, Loi S, Bradbury I, Bliss JM, Azim HA, Jr, Ellis P, Di Leo A, Baselga J, Sotiriou C, PiccartGebhart M. Dissecting the heterogeneity of triplenegative breast cancer. J Clin Oncol. 2012 May 20;30(15):1879-87. DOI:10.1200/JCO.2011.38.2010.

10. Peshkin BN, Alabek ML, Isaacs C. BRCA1/2 mutations and triple negative breast cancers. Breast Dis. 2010;32(1):25-33. DOI:10.3233/BD-2010-0306. 
11. Rosai J. The Breast. In: Rosai and Ackerman's Surgical Pathology. 10 ${ }^{\text {th }}$ Edition (Vol. 2). New York: Mosby (Elsevier);2012.p.1719-20.

12. Shetty P. India faces growing breast cancer epidemic. Lancet. 2012 March 17; 379 (9820):992-3. DOI:http://dx.org/10.1016/S0140-6736(12)60415-2.

13. Al-Mulla F, Abdulrahman M, Varadharaj G, Akther $\mathrm{N}$, Anim JT. BRCA 1 gene expression in breast cancer: a correlative study between real time RT-PC immunohistochemistry. J Histochem Cytochem. 2005 May; 53(5):621-9. DO:10.1369/jhc.4A6544.2005.

14. Burkadze G, Khardzeishvili, Gudadze M, Tsikhiseli G, Turashvili G. Immunohistochemical expression of BRCA 1 protein in invasive ductal carcinoma of breast. Georgian Med News. 2010 July;184:51-60.

15. Lakhani SR, Gusterson BA, Jacquemier J, Sloane JP, Anderson TJ, Vijver MJ, et al. The pathology of familiar breast cancer histological features of cancers in families not attributable to mutations in BRCA1 or BRCA2. Clin Cancer Res. 2000 March;6(3):782-9.

16. Palacios J, Honrado E, Osorio A, Cazorla A, Sarrio D, Barroso A, et al Immunohistochemical Characteristics Defined by Tissue Microarray of Hereditary Breast Cancer Not Attributable to BRCA1 or BRCA2 Mutations: Differences from Breast Carcinomas Arising in BRCA1 and BRCA2 Mutation Carriers. Clin Cancer Res. 2003 Sep1;9(10):3606-14.

17. Atchley DP, Albarracian CT, Lopez A, Valero V, Amos CL, Gonzalez-Angulo AM. Clinical and Pathologic Characteristics of Patients with BRCA Positive and BRCA-Negative Breast Cancer. J Clin Oncol. 2008;26(2):4282-8.

18. Palomba G, Budroni M, Olmeo N, Atzori F, Ionta TM, Pisano $\mathrm{M}$ et al. Triple negative breast cancer frequency and type of BRCA mutation: Clues from Sadinia. Oncol Lett. 2014 April;7(4): 948-52.

19. Mote PA, Leary JA, Avery KA, Sandelin K, Chenevix-Trench G, Kirk JA et al. Germ-line mutations in BRCA1 or BRCA2 in the normal breast are associated with altered expression of estrogenresponsive proteins and the predominance of progesterone receptor A. Genes Chromosomes Cancer. 2004 Mar;39(3):236-48.

20. Veronesi A, de Giacomi C, Magri MD, Lombardi D, Zanetti M, Scuderi C, et al. Familial breast cancer: characteristics and outcome of BRCA 1-2 positive and negative cases. BMC Cancer. 2005 July;5:70. DOI:10.1186/1471-2407-5-70.

\section{How to cite this article?}

Sharma M, Madan M, Manjari M, Madhukar M, Singh T, Gupta S. BRCA1 Positivity in Triple Negative Breast Cancers: A Prospective Study in Tertiary Care Cancer Hospital. Trop J Path Micro 2016;2(2):64-69.doi: 10.17511/jopm.2016.i02.05 